\title{
Imaging Vacancy Defects in Single-layer Chromium Triiodide
}

Jihai Zhang, ${ }^{1,3, \#} Y u$ Guo, ${ }^{2, \#}$ Peigen Li, ${ }^{1,3}$ Jun Wang, ${ }^{1,3}$ Si Zhou, ${ }^{2, *}$ Jijun Zhao, ${ }^{2}$ Donghui Guo, ${ }^{1, *}$ Dingyong Zhong ${ }^{1,3, *}$

\#The authors equally contributed to the work.

${ }^{1}$ School of Physics, Sun Yat-sen University, 510275 Guangzhou, China

${ }^{2}$ Key Laboratory of Materials Modification by Laser, Ion and Electron Beams (Dalian University of Technology), Ministry of Education, 116024 Dalian, China

${ }^{3}$ State Key Laboratory of Optoelectronic Materials and Technologies, Sun Yat-sen University, 510275 Guangzhou, China

${ }^{*}$ Corresponding authors Email:

sizhou@dlut.edu.cn (S.Z.)

guodonghui@mail.sysu.edu.cn (D.G.)

dyzhong@mail.sysu.edu.cn (D.Z.) 


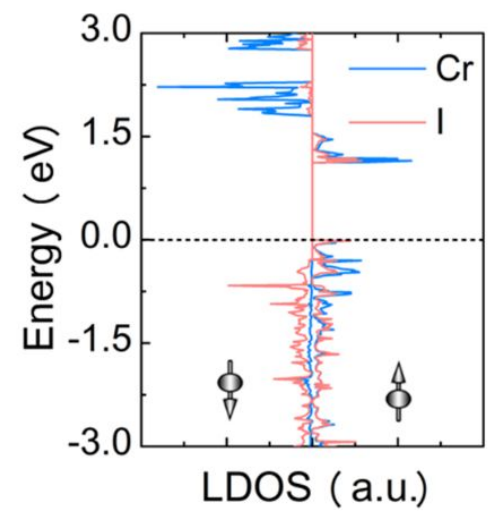

Fig. S1. Local density of states of perfect monolayer $\mathrm{CrI}_{3}$ lattice. 


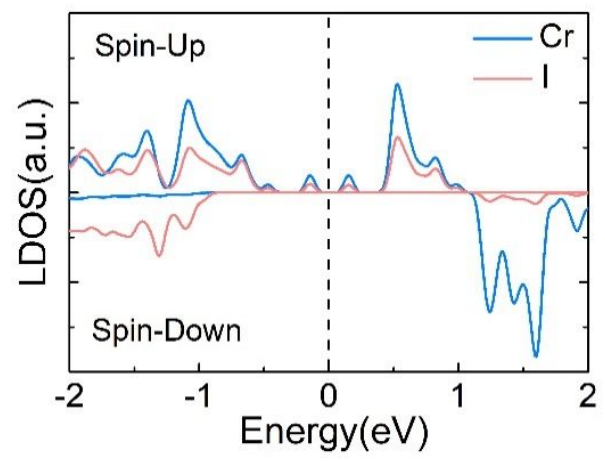

Fig. S2. LDOS for single I vacancy in $\mathrm{CrI}_{3}$ monolayer with smearing value of $0.05 \mathrm{eV}$. 
(a)

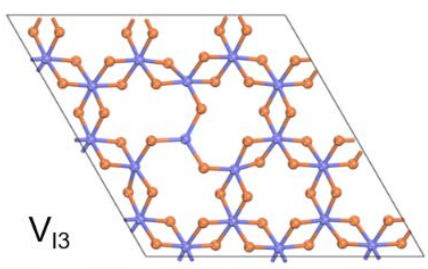

(b)

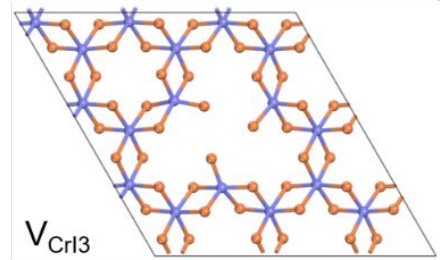

(c)

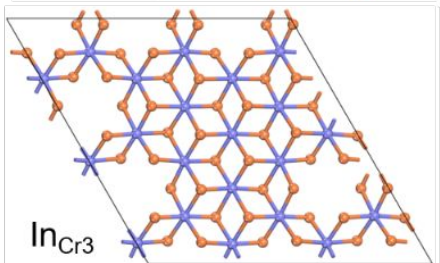

(d)

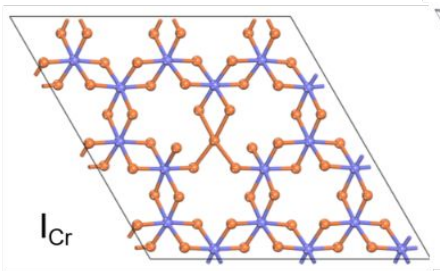

(e)

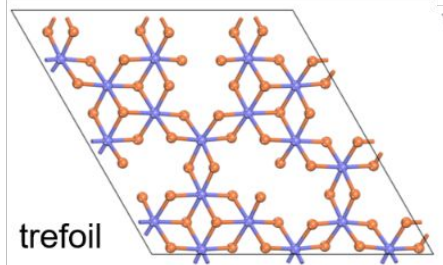

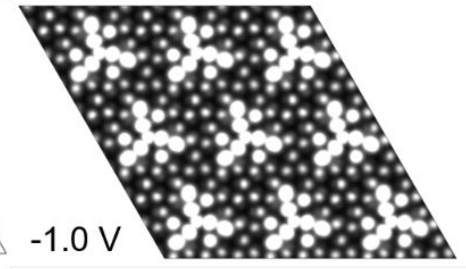

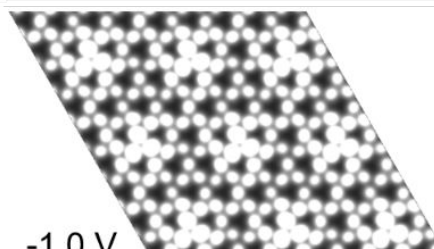

$-1.0 \mathrm{~V}$ toptos
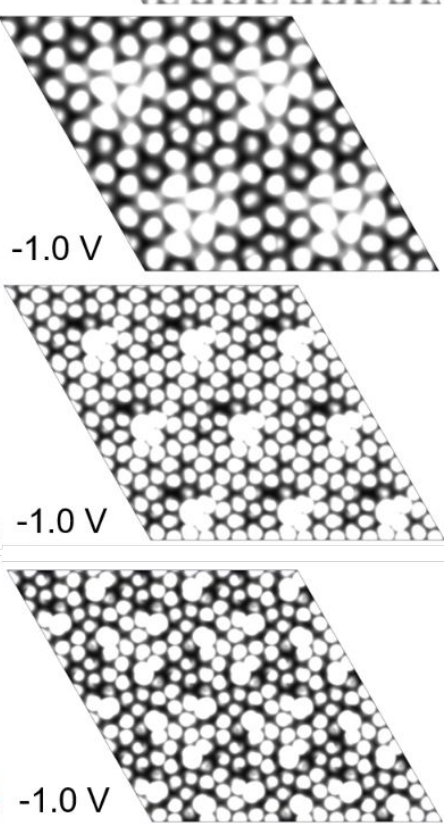

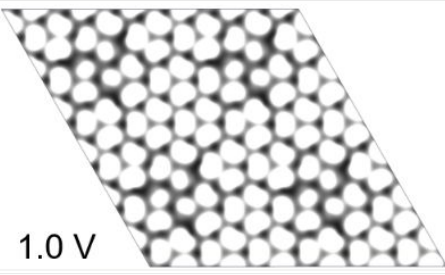

Ho 30 .
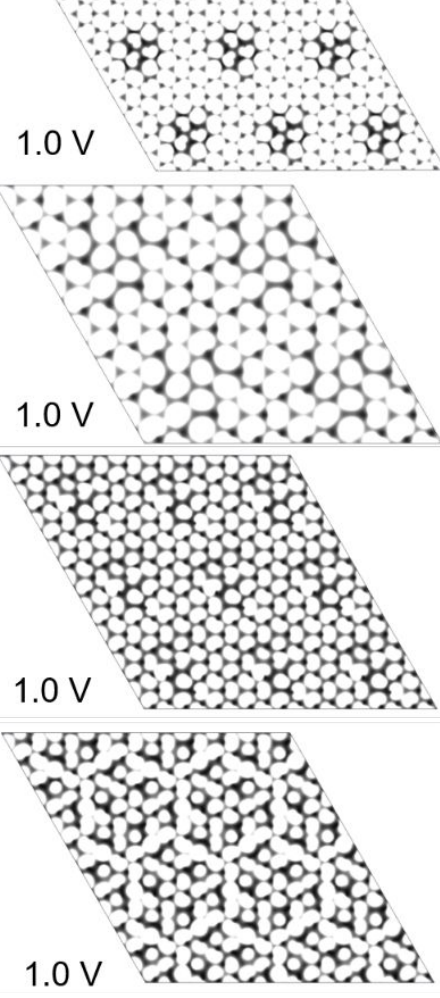

Fig. S3. Optimized structures and STM simulation result of other possible defects. (a)(e) Optimized structures, STM simulation results under $-1.0 \mathrm{~V}$ and $+1.0 \mathrm{~V}$, for three I vacancies $\left(\mathrm{V}_{\mathrm{I} 3}\right), \mathrm{CrI}_{3}$ vacancies $\left(\mathrm{V}_{\mathrm{Cr} 3}\right)$, three insert $\mathrm{Cr}\left(\mathrm{In}_{\mathrm{Cr} 3}\right)$, I anti-site $\left(\mathrm{I}_{\mathrm{Cr}}\right)$, trefoil defects respectively. 
(a)

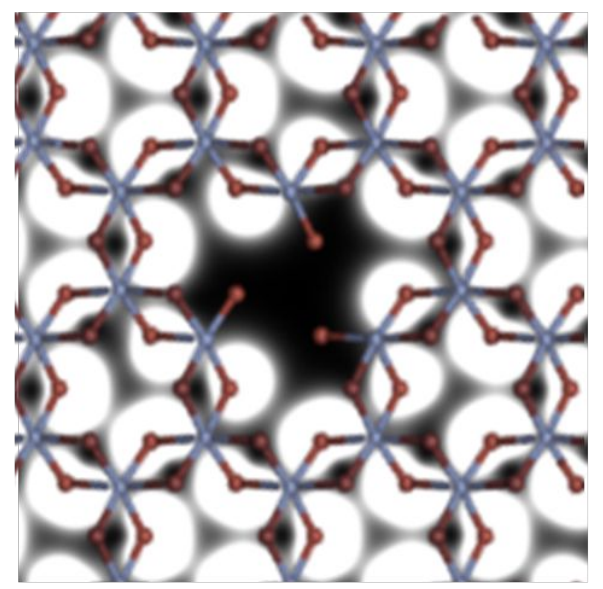

(b)

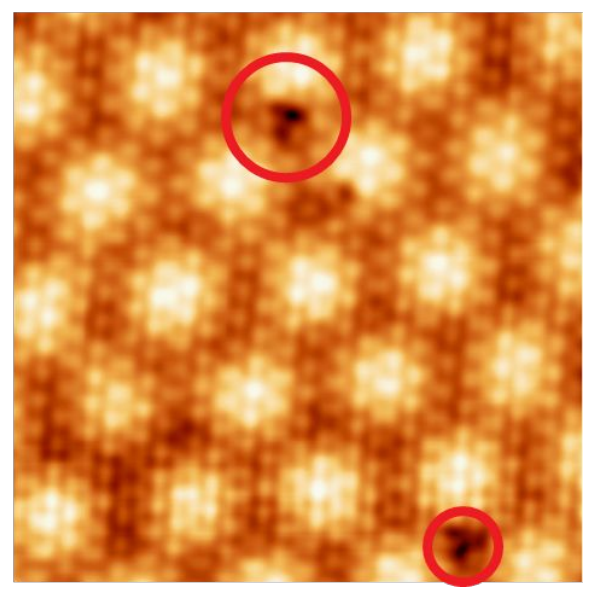

Fig. S4. STM image of upper layer $\mathrm{CrI}_{3}$ vacancies. (a). STM simulation result of the upper layer $\mathrm{CrI}_{3}$ vacancies at $1.0 \mathrm{~V}$. (b) Experimental STM result with similar behavior, $2.0 \mathrm{~V}, 100 \mathrm{pA}$. Considering the migration mechanism for I vacancy, such behavior originates from the upper layer $\mathrm{CrI}_{3}$ vacancies in all possibility. 

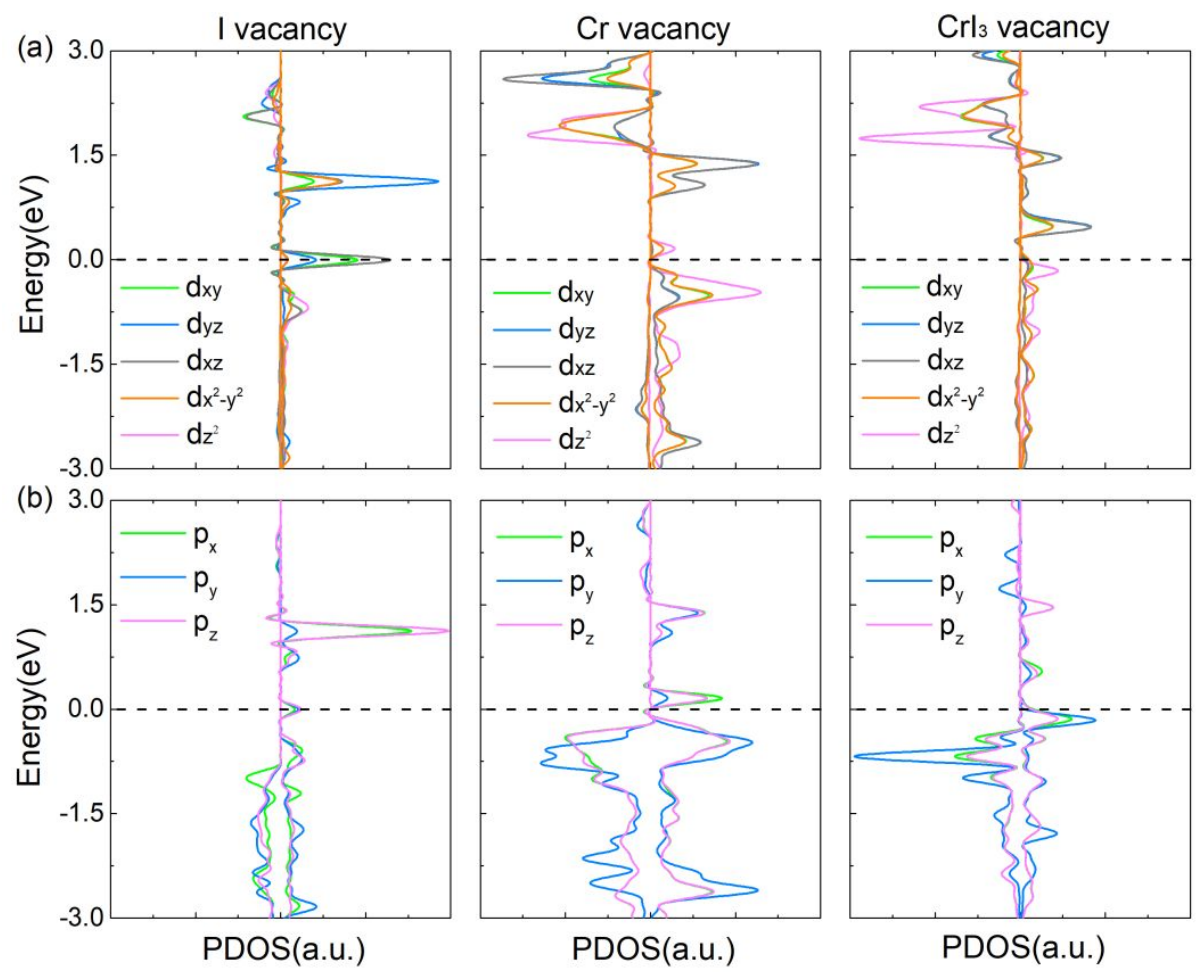

Fig. S5. The projected density of states of (a) $\mathrm{Cr}$ and (b) I atoms around the defects for the $\mathrm{CrI}_{3}$ monolayer (from left to right) with $\mathrm{I}, \mathrm{Cr}$ and $\mathrm{CrI}_{3}$ vacancies, respectively. 
(a)

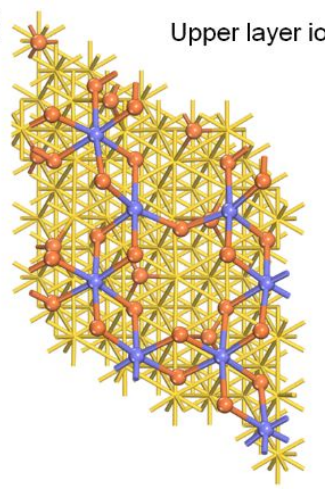

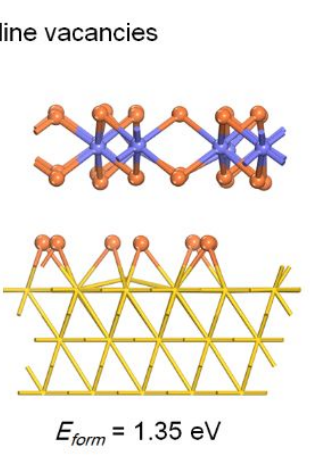

(b)

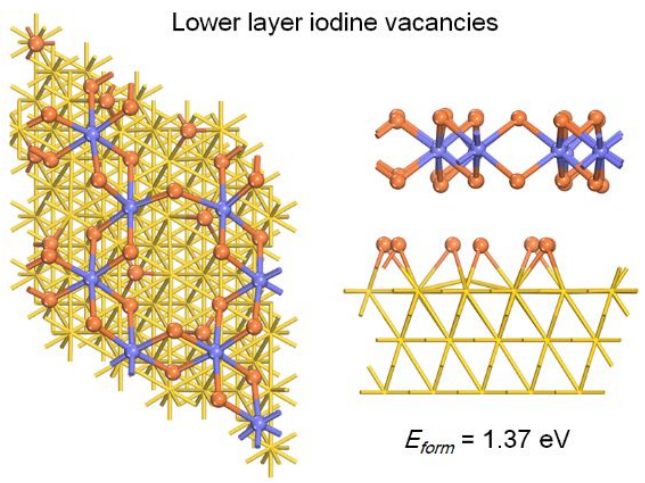

Fig. S6. Formation energy for I vacancies in upper (a) and lower (b) I layers considering the I modified Au (111) substrate. 